\title{
Detection of a Spatially Distributed Target in White Noise
}

\author{
Karl Gerlach, Member, IEEE, Michael Steiner, Member, IEEE, and F. C. Lin
}

\begin{abstract}
A detector of a spatially distributed target in white Gaussian noise is developed. A reasonable distribution for the a priori target scatterer density is assumed, and a detector that incorporates this a priori knowledge is given. A simple detector form results, whose detection performance is robust over different scattering densities.
\end{abstract}

\section{INTRODUCTION}

A $\mathrm{HIGH}$ resolution radar (HRR) can spatially resolve a target into a number of scattering centers depending on the range extent of the target and the range resolution capability of the radar [1]. Range resolution is normally inversely proportional to the radar's transmitted waveform's bandwidth. Radars that are designated as wideband (WB) or ultrawideband (UWB) inherently have a range resolution capability such that a range profile (or spatial distribution) of the amplitude returns from a target can be formed. Normally, the detection of the presence of a target using all of the target's energy found in the range profile is performed first. Thereafter, classification of the target can be attempted using the target's range profile.

For this development, we assume that the radar transmits only one pulse. On receive, this pulse is match filtered (or pulse compressed) so that individual scatterers on a target are resolved. There are a total of $J$ range cells that the target scattering can occur in. Either an in-phase/quadraturephase $(\mathrm{I} / \mathrm{Q})$ or a real representation of the received signal could be used depending on the appropriateness of using either representation. For this development, we choose a real representation. However, we also give the form of the I/Q detector. It is also assumed that additive zero-mean white Gaussian noise is present on each return of the $J$ range cells.

Quite often, some knowledge of the spatial distribution of the desired target is known a priori. For example, it may be known that over the target's range extent, only a small specified number of scattering centers occur. We develop a detection scheme whereby this a priori knowledge can be incorporated. It turns out that a simple detector form results that significantly enhances the detection of a sparsely distributed target. Related research is given in [3]-[7].

Manuscript received October 16, 1996. The associate editor coordinating the review of this manuscript and approving it for publication was Prof. K. Buckley.

The authors are with the Naval Research Laboratory, Washington, DC 20375-5320 USA.

Publisher Item Identifier S 1070-9908(97)05228-0.

\section{Spatial Density DePendent Detector}

In this section, we develop a detector of a spatially distributed target in white noise that incorporates a priori knowledge about the spatial scattering densities of the target scatterers. We assume that the received signal is matched filtered with the transmitted waveform to generate a $J$-length vector $\mathbf{x}$, which represents returns in contiguous range cells across some range extent. The range cell's range width is equal to the radar range resolution. Thus, there are $J$ possible range cells that the target's scatterers can occupy. There would actually be many more than $J$ range cells on receive. We choose to look for a target over a range window of $J$ range cells and assume the target is completely contained within these $J$ range cells. The probability that there are $n$ target scatterers where $n=1,2, \cdots, J$ is denoted by $p_{n}$. We assume that if there are $n$ target scatterers, each scatterer occupies only one of the $J$ possible range cells and any combination of the $n$ scatterers occupying the $J$ range cells is equally likely. Let $\boldsymbol{s}=\left(s_{1}, s_{2}, \cdots, s_{J}\right)$ denote the target's output of the matched filter and $i_{1}, i_{2}, \cdots, i_{n}$ denote the indices of the elements of $s$ (numbered $1,2, \cdots, J$ ), which contain target scattering. Let $\eta$ denote the noise output of the matched filter where $\eta$ is a $J$-length vector.

We wish to find the generalized likelihood ratio test (GLRT) detector for the hypotheses $H_{0}: \boldsymbol{x}=\boldsymbol{\eta}$ (noise only) versus $H_{1}: \boldsymbol{x}=\boldsymbol{s}+\boldsymbol{\eta}$ (signal plus noise). For the GLRT [2], the unknown parameters under each hypothesis are found via maximum likelihood (ML) estimation. Thereafter, the ML estimates under each hypothesis are substituted into their respective probability density functions (pdf's) for each hypothesis. The resultant ratio $\left(H_{1}\right.$ to $\left.H_{0}\right)$ of these altered pdf's is called the GLRT. For the present assume that the noise power, $\sigma^{2}$, is known.

The $H_{1}$ hypothesis is a composite hypothesis since for a given scattering combination, the desired signal elements, $s_{i_{1}}, s_{i_{2}}, \cdots, s_{i_{n}}$, are unknown. The $H_{0}$ hypothesis is simple. If these quantities were known for each scattering combination, the optimal detector is given by

$$
\lambda=\sum_{n=1}^{J} p_{n} \sum_{\Omega_{n}}\left(\begin{array}{l}
J \\
n
\end{array}\right)^{-1} \Lambda\left(s_{i_{1}}, s_{i_{2}}, \cdots, s_{i_{n}}\right)
$$

where $\Omega_{n}$ denotes the set of $n$ out of $J$ scattering combinations, $\left(\begin{array}{l}J \\ n\end{array}\right)$ is the binomial coefficient $J ! /[(J-n) ! n !]$, and $\Lambda\left(s_{i_{1}}, s_{i_{2}}, \cdots, s_{i_{n}}\right)$ is the likelihood ratio of the hypothesis test conditioned on knowing $i_{1}, i_{2}, \cdots, i_{n}$ and $s_{i_{1}}, s_{i_{2}}, \cdots, s_{i_{n}}$. 


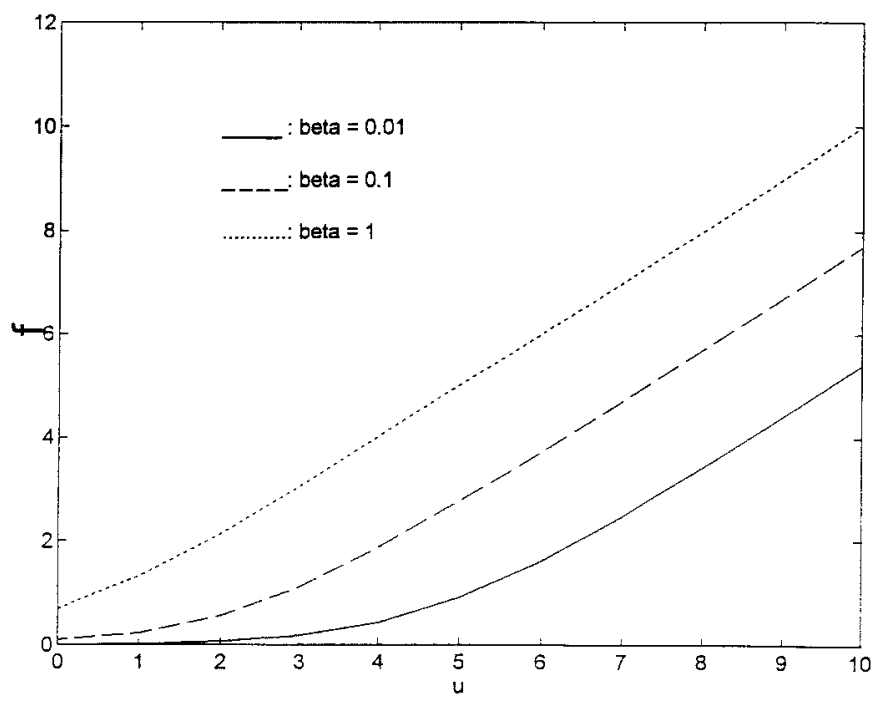

Fig. 1. $f_{\beta}(u)$ versus $u$ and $\beta$.

If the amplitudes of the scatterers are unknown, the GLRT for this detection scheme is found by maximizing $\Lambda\left(s_{i_{1}}, s_{i_{2}}, \cdots, S_{i_{N}}\right)$ over $s_{i_{1}}, s_{i_{2}}, \cdots, s_{i_{n}}$ for each combination of the composite hypothesis. Thus

$$
\begin{aligned}
& \Lambda_{\mathrm{GLRT}}= \\
& \sum_{n=1}^{J} p_{n}\left(\begin{array}{l}
J \\
n
\end{array}\right)^{-1} \sum_{\Omega_{n}} \max _{s_{i_{1}}, s_{i_{2}}, \cdots, s_{i_{n}}} \Lambda\left(s_{i_{1}}, s_{i_{2}}, \cdots, s_{i_{n}}\right) .
\end{aligned}
$$

Since the elements of $\boldsymbol{\eta}$ are independent, identically distributed (i.i.d.) zero-mean Gaussian random variables, it is straightforward to show that

$$
\begin{aligned}
& \Lambda\left(s_{i_{1}}, s_{i_{2}}, \cdots, s_{i_{n}}\right)= \\
& \quad \exp \frac{1}{2 \sigma^{2}}\left[-\sum_{m=1}^{n}\left(x_{i_{m}}-s_{i_{m}}\right)^{2}+\sum_{m=1}^{n} x_{i_{m}}^{2}\right] .
\end{aligned}
$$

Now, $\Lambda(\cdot)$ is maximized with respect to $s_{i_{m}}, m=1, \cdots, n$ when $s_{i_{m}}=x_{i_{m}}$. Thus

$$
\max _{s_{i_{1}}, s_{i_{2}}, \cdots, s_{i_{n}}} \Lambda\left(s_{i_{1}}, s_{i_{2}}, \cdots, s_{i_{n}}\right)=\exp \frac{1}{2 \sigma^{2}} \sum_{m=1}^{n} x_{i_{m}}^{2}
$$

and

$$
\lambda_{\mathrm{GLRT}}=\sum_{n=1}^{J} p_{n} \sum_{\Omega_{n}}\left(\begin{array}{l}
J \\
n
\end{array}\right)^{-1} \exp \frac{1}{2 \sigma^{2}} \sum_{m=1}^{n} x_{i_{m}}^{2} .
$$

We now assume a scattering density for $p_{n}, n=1, \cdots, J$, which has utility in modeling a small number of scatterers and which greatly simplifies the form of the GLRT given by (5). Let

$$
p_{n}=c\left(\begin{array}{l}
J \\
n
\end{array}\right)(1-\alpha)^{j-n} \alpha^{n}, \quad n=1, \cdots, J
$$

where $c=\left[1-(1-\alpha)^{J}\right]^{-1}$ is a constant that satisfies the normalization condition $\Sigma p_{n}=1$ and $\alpha$ is a parameter chosen to control the spatial density of the scatterers. We observe that $p_{n}$ is approximately equal to the binomial distribution with parameter $\alpha$. We choose the parameter $\alpha$ based on the expected number of scatterers, $n_{0}$. For the distribution given by (6), the average number of scatterers is given by $J_{\alpha}\left[1-(1-\alpha)^{J}\right]^{-1}$. Thus, we set

$$
n_{0}=J_{\alpha}\left[1-(1-\alpha)^{J}\right]^{-1}
$$

and solve for $\alpha$. For $n_{0} / J \ll 1, \alpha \approx n_{0} / J$. Hence, for a small number of scatterers relative to $J, \alpha$ will be small. If any scattering combination is equally likely, $\alpha=0.5$, and for a high density of scatterers where $n_{0} \approx J$, then $\alpha \approx 1$.

If $p_{n}$ given by (6) is substituted into (5), we can show that (5) simplifies to the equivalent detector

$$
\lambda_{\mathrm{GLRT}}=c \prod_{n=1}^{J}\left[1-\alpha+\alpha \exp \left(\frac{x_{n}^{2}}{2 \sigma^{2}}\right)\right]-c .
$$

Furthermore, since a monotonically increasing function of the detection statistic is also an equivalent detector, an equivalent GLRT is given by

$$
\lambda_{\mathrm{GLRT}}=\sum_{n=1}^{J} \ln \left[1+\beta \exp \left(\frac{x_{n}^{2}}{2 \sigma^{2}}\right)\right]
$$

where $\beta=\alpha /(1-\alpha)$. It is seen that the spatial scattering density (SSD) GLRT is the sum of identical functions of the normalized power of each range cell return as

$$
\lambda_{\mathrm{GLRT}}=\sum_{n=1}^{J} f_{\beta}\left(u_{n}\right)
$$

where $f_{\beta}(u)=\ln \left(1+\beta e^{u}\right)$ and $u_{n}=x_{n}^{2} / 2 \sigma^{2}$. A plot of $f_{\beta}(u)$ for various values of $\beta$ is given in Fig. 1. It is observed that, as $u$ becomes large, $f_{\beta}(u)$ is asymptotic with the linear function: $u+\ln \beta$. Also, it is seen that the effect of the $\beta$ parameter for sparse scattering $(\beta=0.01,0.1)$ is to suppress the smaller returns. It is speculated that this detector form eases the collapsing loss problems that occur when noise power only range cell returns are summed with signal plus noise power range cell returns in forming the detection statistic. We note that as $\beta \rightarrow \infty$, the detector form becomes

$$
\lambda_{\mathrm{GLRT}}(\beta \rightarrow \infty)=\sum_{n=1}^{J} u_{n}
$$

which is expected because for $\beta \rightarrow \infty$, every range cell is occupied by a scatterer. Thus, in the limit the SSD-GLRT detector is an integrator.

We have assumed that the white noise power level, $\sigma^{2}$, is known. If it were unknown, then it could be estimated by averaging the squared amplitudes of range cells where the target is assumed not to be present. Finally, if the I/Q representation was used for the radar returns (i.e., the elements of $\mathbf{x}$ are complex), then it is straightforward to show that the SSD-GLRT for this representation takes the form

$$
\lambda_{\mathrm{GLRT}}=\sum_{n=1}^{J} \ln \left(1+\beta e^{1 /\left|x_{n}\right|^{2} \sigma^{2}}\right)
$$

where $|\cdot|$ denote complex magnitude. 


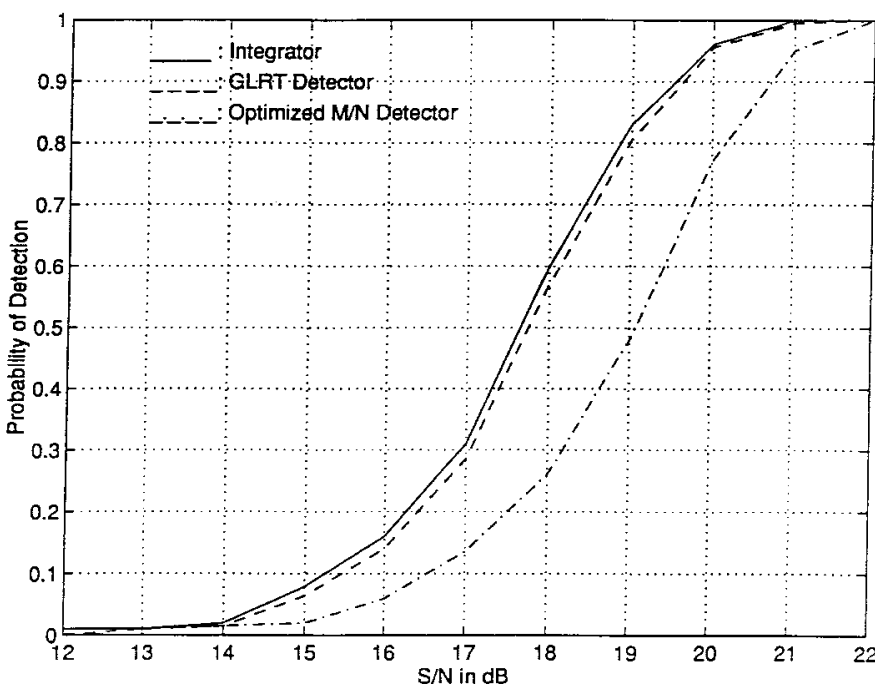

Fig. 2. Comparison of the SSD-GLRT, integrator, and $M$ out of $N$ detector; $J=48, n=48, P_{F}=10^{-6}, M_{C}=1000$

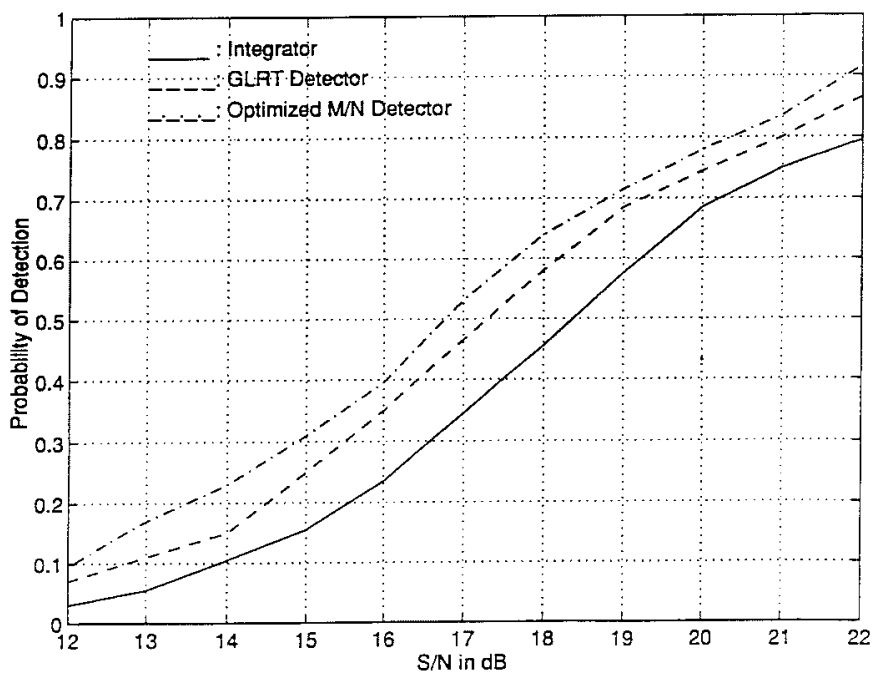

Fig. 3. Comparison of the SSD-GLRT, integrator, and $M$ out of $N$ detector; $J=48, n=2, P_{F}=10^{-6}, M_{c}=1000$.

\section{RESUlTS AND SUMmARY}

In Figs. 2 and 3, we compare the detection performance of the SSD-GLRT with two standard noncoherent detection schemes: an integrator detector and an $M$ out of $N$ detector (sometimes called a binary integrator). The integrator detector has the form given by (9). The signal-to-noise power ratio parameter $(\mathrm{S} / \mathrm{N})$ is defined as ratio of the sum of the signal powers over the $J$ range cells to the noise power per range cell $\left(\sigma^{2}\right)$. The $M$ out of $N$ detector is optimized as follows. For six cases of $\mathrm{S} / \mathrm{N}$ we found the best $M$ for a given $N$. The performance of the $M$ out of $N$ detector for each $\mathrm{S} / \mathrm{N}$ is then plotted with the best $M$. For both figures $J=48$, the probability of false alarm, $P_{F}$, equals $10^{-6}$, and the number of Monte Carlos, $M_{\mathcal{C}}$, used to estimate each detection probability is 1000 . We found the threshold of the test statistic of (5) which achieves the desired $P_{F}$ using Monte Carlo simulation. In Fig. 2, there are $n=48$ scatterers (each cell contains a scatterer) and in Fig. 3, there are $n=2$ scatterers randomly distributed among the $J=48$ range cells. For the SSDGLRT in Fig. 2, we a priori expected equally likely one to 48 scatterers $\left(n_{0}=24\right)$ and, thus, set $\alpha=0.5$ or $\beta=1$. For the SSD-GLRT in Fig. 3, we a priori expected two scatterers $\left(n_{0}=2\right)$ and, thus, set $\alpha=0.04$ or $\beta=0.042$ using (7).

From Fig. 2, in a dense scattering environment we see that the SSD-GLRT significantly out performs the optimized $M$ out of $N$ detector and almost equals the performance of the integrator detector. From Fig. 3, we see that in a sparse scattering environment that the SSD-GLRT out performs the integrator detector while losing about $0.5 \mathrm{~dB}$ in $\mathrm{S} / \mathrm{N}$ performance versus the optimized $M$ out of $N$ detector. Hence, the SSD-GLRT appears to be robust at the two extremes of scattering density. Other performance curves were run (but not presented here), which showed that the SSD-GLRT is robust for many scattering densities.

In summary, we have developed a detector that incorporates knowledge of the scatterer density. We found an SSD-GLRT and compared its performance to conventional $M$ out of $N$ detection and integrator detector. We showed through simulation that the SSD-GLRT is a robust solution when the scattering density parameter is known.

\section{REFERENCES}

[1] D. R. Wehner, High-Resolution Radar, 2nd ed. Boston, MA: Artech House, 1995.

[2] H. L. Van Trees, Detection, Estimation, and Modulation Theory, Part I. New York: Wiley, 1971.

[3] P. K. Hughes II, "A high-resolution radar detection strategy," IEEE Trans. Aerosp. Electron. Syst., vol. AES-19, pp. 663-667, Sept. 1983.

[4] A. Farina and A. Russo, "Radar detection of correlated targets in clutter," IEEE Trans. Aerosp. Electron. Syst., vol. AES-22, pp. 513-532, Sept. 1986.

[5] A. Farina and F. A. Studer, "Detection with high resolution radar: Advanced topics and potential applications," Chin. J. Syst. Eng. Electron., vol. 3, pp. 32-34, 1992.

[6] L. Cai and H. Wang, "Moving target detection performance potential of UWB radars," in Ultrahigh Resolution Radar, Proc. SPIE 1875, R. S. Vickers, Ed., 1993, pp. 2-13.

[7] M. Steiner, K. Gerlach, and F. C. Lin, "Ultrawideband radar detection in white noise," in Ultrawideband Short-Pulse Electromagnetics 3, L. Carin et al., Eds. New York: Plenum, 1997. 